\title{
BMJ Open Impact of the COVID-19 pandemic on the mental health and learning of college and university students: a protocol of systematic review and meta-analysis
}

\author{
Jing Mao, ${ }^{1}$ Xin Gao, ${ }^{2}$ Peireng Yan, ${ }^{3,4}$ Xiaocao Ren, ${ }^{2}$ Yong Guan, ${ }^{5}$ Yi Yan (i) ${ }^{6}$
}

To cite: Mao J, Gao X, Yan $P$, et al. Impact of the COVID-19 pandemic on the mental health and learning of college and university students: a protocol of systematic review and meta-analysis. BMJ Open 2021;11:e046428. doi:10.1136/ bmjopen-2020-046428

- Prepublication history for this paper is available online. To view these files, please visit the journal online (http://dx.doi. org/10.1136/bmjopen-2020046428).

$\mathrm{JM}, \mathrm{XG}$ and PY are joint first authors.

Received 04 November 2020 Accepted 07 July 2021

Check for updates

(C) Author(s) (or their employer(s)) 2021. Re-use permitted under CC BY-NC. No commercial re-use. See rights and permissions. Published by BMJ.

For numbered affiliations see end of article.

Correspondence to

Dr Yi Yan;

15002665669@139.com

\section{ABSTRACT}

Introduction COVID-19 has a serious impact on people's physical health and mental health. The COVID-19 pandemic forced college and university students to take online classes, which may have bad impacts on students' learning. In addition, the students lost many job opportunities during the pandemic. Faced with employment and study pressure and worried about the epidemic, college and university students were prone to increased overall negative emotion, anxiety and depression. Therefore, this systematic review and metaanalysis will be conducted to explore the impact of the COVID-19 pandemic on the mental health and learning of college and university students.

Methods and analysis We will conduct electronic literature search of the PubMed, Embase, Cochrane Library, Web of Science and Chinese National Knowledge Infrastructure databases. Two researchers will independently screen the studies, extract data and assess the quality of the included studies. Any disagreement will be resolved by the third investigator. The NewcastleOttawa Scale and other tools will be used to assess the risk of bias, according to the study design of included studies. OR, risk ratio, mean difference and $95 \% \mathrm{Cl}$ will be considered as the effect size. Heterogeneity between studies will be assessed by subgroup and sensitivity analysis, and publication bias will be detected by funnel plots, Begg's test and Egger's test.

Ethics and dissemination This systematic review and meta-analysis involves no patient contact and no interaction with healthcare providers or systems. We will disseminate the findings of this study through the presentation at scientific conferences and publication in a peer-reviewed journal.

PROSPERO registration number CRD42020201132.

\section{INTRODUCTION}

COVID-19 is a newly discovered infectious disease caused by the SARS-CoV-2. ${ }^{1}$ COVID-19 has been classified as a pandemic by the WHO on 11 March $2020 .^{2}$ The WHO reported, as of 28 October 2020, there were almost 43.5 million confirmed COVID-19 cases, including more than 1 million deaths. ${ }^{3}$ In the past 2 months, the number of new cases per week has remained more than 2 million. ${ }^{4}$

\section{Strengths and limitations of this study}

- This study will be the first synthesis of research into the impact of COVID-19 pandemic on the mental health and learning of college and university students.

- The study selection, data extraction and risk of bias evaluation will be performed by two independent reviewers to minimise the potential bias.

- This study will explore the differences in the impact of the COVID-19 pandemic between various students.

- Only the studies published in English or Chinese will be included because of the limited resource and the authors' linguistic competence.

- The different assessment scales of mental health will be a major source of heterogeneity for this study.

With various transmitted modes, ${ }^{5}$ COVID-19 has a serious impact on people's physical health. People infected by this virus may be asymptomatic, or they may have six different 'types' of symptoms. ${ }^{6}$ The main symptoms of COVID-19 were the respiratory and digestive system symptoms, ${ }^{7}$ severe cases may lead to respiratory failure, cardiac arrest and death. ${ }^{8}$

Additionally, COVID-19 also has a serious impact on people's mental health. During the COVID-19 pandemic, people suffer from the wider social impact of the pandemic, the governmental response, unemployment, financial difficulties, and other experiences such as concern about their illness, stigma, amnesia or traumatic memories of severe illness. ${ }^{9}$ Several systematic reviews and meta-analyses suggested an important impact of the COVID-19 outbreak on the general population's mental health ${ }^{9-12}$ and in specific population. ${ }^{13-15}$ Therefore, both the infected and non-infected population were under tremendous psychological pressure. ${ }^{16}$

College and university students should maintain a good attitude and work hard to learn knowledge, adapting to social life 
in advance and striving for the future. However, the measures to prevent the further spread of COVID-19 made the lifestyle and learning of college and university students changed. Moreover, the changes made the students prone to anxiety and irritability. ${ }^{17} 18$ Moreover, some students were prone to have mental health problems because of the pressure of employment and entrance examinations. ${ }^{19}$ Additionally, research found that those aged 21-40 years old and with higher education level were more likely to be anxious and depressed during the COVID-19 pandemic. ${ }^{20}{ }^{21}$ Recently, several surveys research into the mental health of college students during the COVID-19 pandemic and found there is a high prevalence of anxiety, depression and other mental health problems among university and college students, ${ }^{22-25}$ they are even at higher risk of experiencing mental health problems than other populations. ${ }^{2627}$

On the other hand, the mental health of college and university students will affect the attention, energy and motivation of students. All these had a vital impact on the college and university students' learning and ability to obtain good higher education. ${ }^{28} 29$

A high-quality meta-analysis has been increasingly regarded as one of the key tools for achieving evidence. But there was no systematic review and meta-analysis on the impact of the COVID-19 pandemic on the mental health and learning of college and university students. Therefore, this systematic review and meta-analysis will be conducted to identify the impact of the COVID-19 pandemic on the mental health and learning of college and university students.

\section{Review question}

What is the impact of the COVID-19 pandemic on the mental health and learning of college and university students?

\section{METHODS}

The Preferred Reporting Items for Systematic Reviews and Meta-Analysis Protocols guideline was used to format this protocol. ${ }^{30}$

\section{Patient and public involvement}

No patient involved. No requirement of ethical approval and informed consent is needed because it is a retrospective study.

\section{Search strategy}

We will conduct electronic literature search of the PubMed, Embase, Cochrane Library, Web of Science and Chinese National Knowledge Infrastructure databases. There are no time and languages restrictions. The search strategy will include key words relating to COVID-19 and mental health. The keywords will be used and a systematic literature search will be carried out based on the following keywords: ("Students"[Mesh]) AND ( ( ( ( ( ( ( ("severe acute respiratory syndrome coronavirus 2" [Supplementary Concept])
OR (2019-nCoV[Title/Abstract])) OR (SARS-CoV2[Title/Abstract])) OR (2019 novel coronavirus[Title/ Abstract])) OR (COVID-19 virus[Title/Abstract])) OR (coronavirus disease 2019 virus[Title/Abstract])) OR (COVID-19 virus[Title/Abstract])) OR ((“COVID-19” [Supplementary Concept] $)$ OR $((()((()((2019$ novel coronavirus disease[Title/Abstract]) OR (COVID19[Title/Abstract])) OR (COVID-19 pandemic[Title/ Abstract])) OR (SARS-CoV-2 infection[Title/Abstract])) OR (COVID-19 virus disease[Title/Abstract])) OR (2019 novel coronavirus infection[Title/Abstract])) OR (2019$\mathrm{nCoV}$ infection[Title/Abstract])) OR (coronavirus disease 2019[Title/Abstract])) OR (coronavirus disease19[Title/Abstract])) OR (2019-nCoV disease[Title/ Abstract])) OR (COVID-19 virus infection[Title/ Abstract])))). References of included studies will also be traced back to find potential qualified studies.

\section{Inclusion criteria}

We will include all peer-reviewed articles exploring mental health and learning problems of college and university students during the COVID-19 pandemic.

\section{Types of participants/population}

College and university students exposed to the COVID-19 pandemic, with no age range, gender, ethnicity or health status restrictions.

\section{Types of study designs}

We will include all peer-reviewed primary studies that use any study design (eg, randomised trial, cohort, casecontrol, longitudinal, cross-sectional, etc).

\section{Types of outcomes}

The mental health of college and university students during the COVID-19 pandemic (eg, depression, anxiety, post-injury stress disorder, suicide or suicidal ideation, fatigue and laziness, and psychological interventions). We also choose the outcomes about the impact of the COVID-19 pandemic on the learning of college and university students.

\section{Exclusion criteria}

Studies will be excluded if one of the following conditions is met: (1) the research data are not complete; (2) qualitative studies, editors, letters, comments, notes, reviews and meta-analyses; (3) the study is not in Chinese or English.

\section{Study selection}

Two researchers will independently screen the studies retrieved during the searches to identify those that may meet the inclusion criteria, and to exclude irrelevant studies. Any conflicts arise during the screening of the articles will be resolved by the third researcher. Titles and abstracts of the studies retrieved by the literature search will be screened based on inclusion/exclusion criteria, and we will acquire the full text of potentially relevant studies for further assessment. 


\section{Data extraction}

A standard form will be used to extract data from the included studies. Two reviewers will perform the data extraction independently. Discrepancies between the reviewers will be resolved either by discussion or by a third reviewer. To identify other relevant study data, we will contact the authors of published studies for incomplete data.

Information to be extracted includes four domains: (1) study characteristics (authors, year of publication, country, date of data collection); (2) participant information (age, gender, ethnicity, health status, institutions of higher education and other characteristics such as infected/not infected with COVID-19); (3) methods (study design, measures, validity, reliability); and (4) any other information including COVID-19-related information about the study that is regarded as necessary.

\section{Risk of bias evaluation}

Two researchers will independently assess the risk of bias of included studies. If there is any question, a third investigator will solve it through discussion. The Newcastle-Ottawa Scale $^{31}$ and other tools will be used to assess the risk of bias, according to the study design of included studies.

\section{Data synthesis and meta-analysis}

The available evidence will be synthesised in a metaanalysis to derive a pool estimate of the problems of mental health and learning for college and university students during COVID-19. This is dependent on enough relevant studies for a meta-analysis.

In this meta-analysis, OR, risk ratio and $95 \%$ CI will be considered as the effect size for dichotomous outcomes, weighted mean difference or standardised mean difference with $95 \%$ CI will be calculated as the effect size for continuous outcomes. Forest plots will be produced to visually assess the effect size and corresponding 95\% CI using random-effects models.

Heterogeneity between studies will be assessed via the forest plot, while $\mathrm{I}^{2}$ values described the total variation between studies. $\mathrm{I}^{2}$ values of $<25 \%, 25 \%-50 \%$, and $>50 \%$ indicate low, moderate, and high heterogeneity, respectively. $\mathrm{P}$ values for heterogeneity were derived using the Cochran Q statistic.

Funnel plots, Begg's test and Egger's test ${ }^{32}$ would be adopted to detect publication bias only when there are at least 10 studies reporting the primary outcomes. All statistical analyses were performed with STATA V.15.

We will perform subgroup analyses using the following group variables: (1) the health of college and university students (college and university students infected by 2019-nCoV; identified as suspected patients or in close contact with patients with 2019-nCoV; general college and university students, etc); and (2) the type of college and university students: medical students, overseas students or exchange student, and other students. We will carry out a sensitivity analysis by excluding one study each time to explore whether the results were driven by a study with an extreme result. We will also perform meta-regression to explore the sources of heterogeneity when there are not fewer than 10 studies in a meta-analysis. ${ }^{33}$

\section{Current status and plan}

The review is under preliminary searches, and is expected to be completed on January 2022.

\section{Ethics and dissemination}

This systematic review and meta-analysis involves no patient contact and no interaction with healthcare providers or systems. We will disseminate the findings of this study through the presentation at scientific conferences and publication in a peer-reviewed journal.

\section{DISCUSSION}

COVID-19 also has a serious impact on people's physical health. Several systematic reviews and meta-analyses suggested the COVID-19 outbreak had an important impact on people's mental health. ${ }^{9-15}$ Facing the pressure of study, employment, great changes of life and worried about the COVID-19 epidemic, college and university students were prone to negative emotions. ${ }^{34}$ Moreover, college and university students were a high-risk population in mental health problems for the age and higher education level. ${ }^{35}$ The mental health problems and the COVID-19 pandemic had an impact on the learning of college and university students. We conducted this systematic review and meta-analysis to provide evidence on the impact of the COVID-19 pandemic on college and university students' mental health and learning.

However, there may be some limitations for this research. First, the mental health status of the students before the COVID-19 pandemic, economic status and other factors have influences on the mental health and learning during the pandemic, but we cannot do the subgroup analysis of these factors for the insufficient data. Second, only the studies published in English or Chinese will be included because of the limited resource and the authors' linguistic competence. Additionally, the use of different scales to assess mental health may be a major source of heterogeneity in this study.

In summary, this systematic review and meta-analysis will provide evidence on the impact of the COVID-19 pandemic on the mental health and learning of college and university students. The final report of this study in the form of scientific paper will be published in a peerreviewed journal. It is expected that the findings of this review provide evidence for psychiatrists/psychologists, public health and education specialists, policymakers and others working in public health emergencies.

\section{Author affiliations}

${ }^{1}$ Department of Mental Health, Lanzhou University Second Hospital, Lanzhou, Gansu, China

${ }^{2}$ The Second Clinical Medical College of Lanzhou University, Lanzhou, Gansu, China ${ }^{3}$ Gansu province key laboratory of groundwater engineering and geothermal resources, Geological Environmental Monitoring Institute of Gansu Province, Lanzhou, Gansu, China 
${ }^{4}$ Environmental Geology and Disaster Prevention and Control of Engineering Technology Research Center in Gansu Province, Geological Environment Monitoring Institute of Gansu Province, Lanzhou, Gansu, China

${ }^{5}$ The First Clinical Medical College of Lanzhou University, Lanzhou, Gansu, China ${ }^{6}$ Department of Project Management Office, Lanzhou University Second Hospital, Lanzhou, Gansu, China

Contributors Conceptualisation-YY and JM. Data curation-JM, XG and PY. Formal analysis—PY and XG. Investigation-JM, XG, PY and YG. Methodology-YY, $J M, X G$ and PY. Project administration-YY and JM. Software-JM, XG, PY and XR. Supervision - YY and JM. Writing (original draft)—JM, XG and PY. Writing (review and editing)—YY, JM, XG, PY, XR and YG.

Funding This study received fundings from the science and technology department of Gansu Province (project grant number: 20CX9ZA113); the Health Industry Plan Management Project of Gansu Province(project grant number: GSWSKY2020-58).

Competing interests None declared.

Patient and public involvement Patients and/or the public were not involved in the design, or conduct, or reporting, or dissemination plans of this research.

Patient consent for publication Not required.

Provenance and peer review Not commissioned; externally peer reviewed.

Open access This is an open access article distributed in accordance with the Creative Commons Attribution Non Commercial (CC BY-NC 4.0) license, which permits others to distribute, remix, adapt, build upon this work non-commercially, and license their derivative works on different terms, provided the original work is properly cited, appropriate credit is given, any changes made indicated, and the use is non-commercial. See: http://creativecommons.org/licenses/by-nc/4.0/.

ORCID iD

Yi Yan http://orcid.org/0000-0002-1539-4087

\section{REFERENCES}

1 Dömling A, Gao L. Chemistry and biology of SARS-CoV-2. Chem 2020;6:1283-95.

2 Espinosa OA, Zanetti ADS, Antunes EF, et al. Prevalence of comorbidities in patients and mortality cases affected by SARSCoV2: a systematic review and meta-analysis. Rev Inst Med Trop Sao Paulo 2020;62:e43.

3 World Health Organization. Coronavirus disease (COVID-19) pandemic: World Health Organization, 2020. Available: https://www. who.int/emergencies/diseases/novel-coronavirus-2019 [Accessed 27 Oct 2020].

4 World Health Organization. WHO Coronavirus Disease (COVID-19) Dashboard: World Health Organization, 2020. Available: https:// covid19.who.int/ [Accessed 27 Oct 2020].

5 Yu ITS, Li Y, Wong TW, et al. Evidence of airborne transmission of the severe acute respiratory syndrome virus. N Engl J Med 2004;350:1731-9.

6 Wise J. Covid-19: study reveals six clusters of symptoms that could be used as a clinical prediction tool. BMJ 2020;370:m2911.

7 Chen N, Zhou M, Dong X, et al. Epidemiological and clinical characteristics of 99 cases of 2019 novel coronavirus pneumonia in Wuhan, China: a descriptive study. Lancet 2020;395:507-13.

8 Denault AY, Delisle S, Canty D, et al. A proposed lung ultrasound and phenotypic algorithm for the care of COVID-19 patients with acute respiratory failure. Can J Anaesth 2020;67:1393-404.

9 Rogers JP, Chesney E, Oliver D, et al. Psychiatric and neuropsychiatric presentations associated with severe coronavirus infections: a systematic review and meta-analysis with comparison to the COVID-19 pandemic. Lancet Psychiatry 2020;7:611-27.

10 Bueno-Notivol J, Gracia-García P, Olaya B, et al. Prevalence of depression during the COVID-19 outbreak: a meta-analysis of community-based studies. Int J Clin Health Psychol 2021;21:100196.

11 Santabárbara J, Lasheras I, Lipnicki DM, et al. Prevalence of anxiety in the COVID-19 pandemic: an updated meta-analysis of communitybased studies. Prog Neuropsychopharmacol Biol Psychiatry 2021;109:110207.

12 Hessami K, Romanelli C, Chiurazzi M. COVID-19 pandemic and maternal mental health: a systematic review and meta-analysis. $J$ Matern Fetal Neonatal Med 2020:1-8.
13 Yan H, Ding Y, Guo W. Mental health of pregnant and postpartum women during the coronavirus disease 2019 pandemic: a systematic review and meta-analysis. Front Psychol 2020;11:617001.

14 Salazar de Pablo G, Vaquerizo-Serrano J, Catalan A, et al. Impact of coronavirus syndromes on physical and mental health of health care workers: systematic review and meta-analysis. J Affect Disord 2020;275:48-57.

15 Lasheras I, Gracia-García P, Lipnicki D, et al. Prevalence of anxiety in medical students during the COVID-19 pandemic: a rapid systematic review with meta-analysis. Int J Environ Res Public Health 2020;17:6603.

16 Rehman S, Lela U. Psychological aid to COVID-19 pandemic: a mental health response to crises management. Psychiatr Danub 2020;32:262-5

17 Walke HT, Honein MA, Redfield RR. Preventing and responding to COVID-19 on College Campuses. JAMA 2020;324:1727.

18 Wilson E, Donovan CV, Campbell M, et al. Multiple COVID-19 Clusters on a University Campus - North Carolina, August 2020. MMWR Morb Mortal Wkly Rep 2020;69:1416-8.

19 Zhang $\mathrm{Y}$, Zhang $\mathrm{H}$, Ma X, et al. Mental health problems during the COVID-19 pandemics and the mitigation effects of exercise: a longitudinal study of college students in China. Int $J$ Environ Res Public Health 2020;17. doi:10.3390/ijerph17103722. [Epub ahead of print: 2505 2020].

20 Zheng Z, Peng F, Xu B, et al. Risk factors of critical \& mortal COVID-19 cases: A systematic literature review and meta-analysis. $J$ Infect 2020;81:e16-25.

21 Ahmed MZ, Ahmed O, Aibao Z, et al. Epidemic of COVID-19 in China and associated psychological problems. Asian J Psychiatr 2020;51:102092.

22 Wang X, Hegde S, Son C, et al. Investigating mental health of US college students during the COVID-19 pandemic: cross-sectional survey study. J Med Internet Res 2020;22:e22817.

23 Dhar BK, Ayittey FK, Sarkar SM. Impact of COVID-19 on psychology among the university students. Global Challenges 2020;4:2000038.

24 Debowska A, Horeczy B, Boduszek D, et al. A repeated crosssectional survey assessing university students' stress, depression, anxiety, and suicidality in the early stages of the COVID-19 pandemic in Poland. Psychol Med 2020:1-4.

25 Ghazawy ER, Ewis AA, Mahfouz EM, et al. Psychological impacts of COVID-19 pandemic on the university students in Egypt. Health Promot Int 2020;37. doi:10.1093/heapro/daaa147. [Epub ahead of print: 25 Dec 2020].

26 Rogowska AM, Pavlova I, Kuśnierz C, et al. Does physical activity matter for the mental health of university students during the COVID-19 pandemic? J Clin Med 2020;9. doi:10.3390/jcm9113494. [Epub ahead of print: 2910 2020].

27 Solomou I, Constantinidou F. Prevalence and predictors of anxiety and depression symptoms during the COVID-19 pandemic and compliance with precautionary measures: age and sex matter. Int J Environ Res Public Health 2020;17. doi:10.3390/ijerph17144924. [Epub ahead of print: 0807 2020].

28 Arenas DL, Viduani AC, Bassols AMS, et al. Peer support intervention as a tool to address college students' mental health amidst the COVID-19 pandemic. Int J Soc Psychiatry 2021;67:301-2

29 Andrews JL, Foulkes L, Blakemore S-J. Peer influence in adolescence: public-health implications for COVID-19. Trends Cogn Sci 2020;24:585-7

30 Moher D, Shamseer L, Clarke M, et al. Preferred reporting items for systematic review and meta-analysis protocols (PRISMA-P) 2015 statement. Syst Rev 2015;4:1.

31 et alWells BS, D GA, O'Connell JP, Welch V. The Newcastle-Ottawa scale (NOS) for assessing the quality of nonrandomised studies in meta-analyses, 2019. Available: http://www.ohri.ca/programs/ clinical_epidemiology/oxford.asp [Accessed 23 Oct 2020].

32 Irwig L, Macaskill P, Berry G, et al. Bias in meta-analysis detected by a simple, graphical test. Graphical test is itself biased. BMJ 1998;316:470.

33 Deeks JJ, Higgins JP, Altman DG. Cochrane Handbook for Systematic Reviews of Interventions. Version 6.2 [updated 2021]. 2021.

34 Liang L, Ren H, Cao R, et al. The effect of COVID-19 on youth mental health. Psychiatr Q 2020;91:841-52.

35 Son C, Hegde S, Smith A, et al. Effects of COVID-19 on college students' mental health in the United States: interview survey study. J Med Internet Res 2020;22:e21279. 